Research Article

\title{
Research on New Types of Suspension Vibration Reduction Systems (SVRSs) with Geometric Nonlinear Damping
}

\author{
Meng Yang $(\mathbb{D}$, Jian Zhang $(\mathbb{D}$, and Xingjiu Luo $\mathbb{D}$ \\ Naval Medical Center of PLA, Shanghai 200433, China \\ Correspondence should be addressed to Jian Zhang; tsyxzx19@163.com
}

Received 25 November 2020; Revised 2 February 2021; Accepted 27 March 2021; Published 8 April 2021

Academic Editor: Shuaishuai Sun

Copyright (c) 2021 Meng Yang et al. This is an open access article distributed under the Creative Commons Attribution License, which permits unrestricted use, distribution, and reproduction in any medium, provided the original work is properly cited.

Firstly, this paper puts forward two new types of suspension vibration reduction systems (SVRSs) with geometric nonlinear damping based on general SVRS (GSVRS), which only has geometric nonlinear stiffness. Secondly, it derives the motion differential equations for the two new types of SVRS, respectively, and discusses the similarities and differences among the two types and GSVRS through the comparison of motion differential equations. Then, it conducts dimensionless processing of the motion differential equations for the two new types of SVRS and carries out a comparative study on the vibration isolation performance of the two types of SVRS under impact excitation and random excitation, respectively. At last, it performs the optimal computation of the chosen new type of SVRS through the ergodic optimization method and studies the influence rule of SVRS parameters on vibration isolation performance so as to realize the optimization of vibration isolation performance.

\section{Foreword}

Product damage and function failure caused by improper packing often occur in existing logistics transportation system, and main factors of the damage refer to vibration and impact in logistics transportation, such as high/low frequency vibration of products during the transportation by highway, railway, sea and air, and drop impact in handling process. In order to inhibit the influence of vibration on the transported goods effectively, it is required to take reasonable vibration reduction measures to protect the transported goods; and SVRS is widely used in numerous cushion packaging technologies.

GSVRS takes spring as the damping element to suspend the vibrated object inside the vibration reduction system, as shown in Figure 1. It is especially suitable for the vibration isolation of precision instruments and equipment with low fragilities, such as large electronic tube and guidance device, which can provide cushion protection for the vibrated object in many directions and can be realized easily. Although SVRS has many advantages, the research on it is scanty and incomplete at present. Zhang [1] studied the vibration reduction performance of diagonal spring early. Then, Xu [2] conducted a preliminary study on the vibration and impact characteristics of SVRS. Wu [3,4] et al. discussed the natural vibration of SVRS and the vibration performance under the effect of foundation displacement. Wang $[5,6]$ et al. studied the vibration performance of SVRS under impact excitation and rectangular pulse excitation. Song $[7,8]$ et al. conducted the research on drop impact response of SVRS by variational iteration method. Zhao $[9,10]$ et al. developed single-dof SVRS into 2-dof SVRS and 3-dof SVRS, respectively, in consideration of elasticity and rotation of the vulnerable part and studied their vibration reduction performance and shock spectrum characteristics. In addition, Kong [11] and Duan [12], respectively, carried out detailed research on the vibration characteristics of vibration reduction systems with the same diagonal spring under sine pulse excitation and rectangular pulse excitation. The studies above deepen the understanding of SVRS; however, it can be found they have obvious limitations. Firstly, all of the studies focus on the discussion about geometric nonlinear stiffness rather than geometric nonlinear damping of SVRS, while all of the elastic materials used in practice have damping inevitably, and geometric nonlinear damping (parallel with suspension stiffness) introduced to SVRS will make the SVRS more 


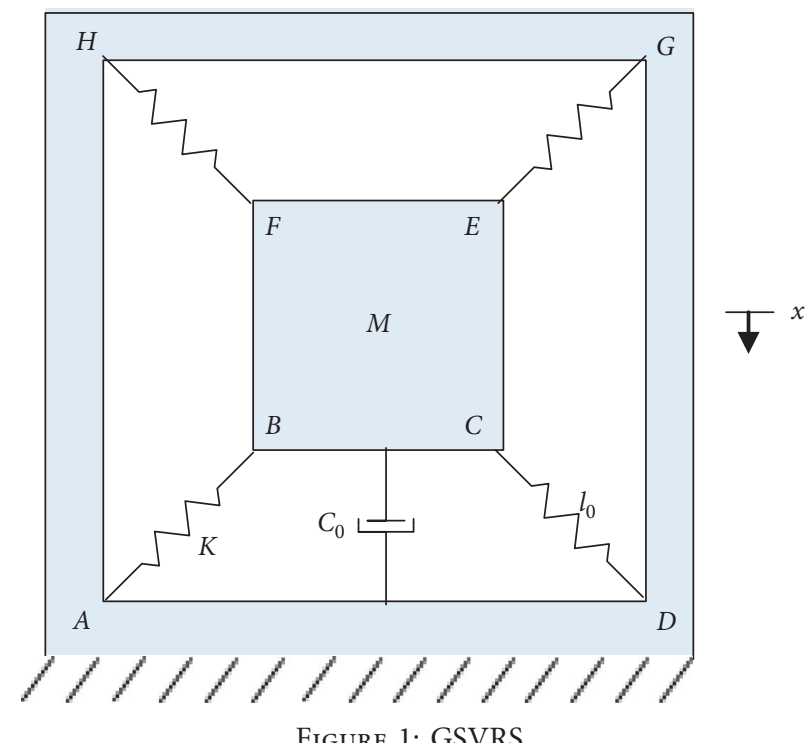

practical; secondly, all of the studies were completed under impact excitation, although impact excitation may cause severe damage to the vibrated object, it seldom happens in transportation, while random excitation is throughout the entire transportation, thus it is of universal significance to study the vibration characteristics of SVRS under random excitation.

In order to better understand the geometric nonlinear damping mentioned above, some related studies [13-17] are introduced. Their geometric nonlinear damping force is mainly produced by horizontal damping. According to these studies, the geometric nonlinear damping force is not only related to the speed but also to the displacement or the higher power of displacement, which will make the geometric nonlinear damping force show more complex characteristics. This will be the difficulty of study.

Based on the analysis above, this paper will put forward two new types of SVRS with geometric nonlinear damping based on GSVRS and compare them with GSVRS to discuss the similarities and differences among the three types. Then, it will carry out a comparative study on the vibration isolation performance of the two new types of SVRS under impact excitation and random excitation, respectively, so as to choose the optimal design scheme. At last, it will optimize the chosen new type of SVRS through ergodic optimization so as to realize the optimization of vibration isolation performance. Through the research, this paper will provide a new thought for the design of SVRS.

\section{Establishment of Motion Equation for the New Types of SVRS}

Figure 1 shows the theoretical model of GSVRS, and diagonal linear damping and lateral linear damping are, respectively, added between ADGH and BCEF to constitute new SVRS, as shown in Figure 2. Type I refers to the new SVRS with diagonal damping, while type II refers to the new SVRS with lateral damping. The motion differential equations for the two new types of SVRS are, respectively, established as below.
2.1. Establishment of Motion Differential Equation for Type I. There are, respectively, four springs and dampers on the top and at the bottom of Type I to support the vibrated object diagonally, which have the same stiffness coefficient, damping coefficient, and original length. The position shown in the figure is the one where deformation has not occurred to the spring and damper. The original length is as follows $\mathrm{HF}=\mathrm{GE}=\mathrm{AB}=\mathrm{DC}=10$. The included angle with horizontal direction is as follows: $\angle \mathrm{GHF}=\angle \mathrm{HGE}=\angle \mathrm{DAB}=\angle \mathrm{ADC}=\varphi_{0}$. The diagonal stiffness is $\mathrm{K}$, and the diagonal damping is $C 1$. Meanwhile, it is considered that the vertical damping is $\mathrm{CO}$, and the mass of the vibrated object is $M$. When the SVRS reaches equilibrium under the action of weight, the gravity center of the vibrated object moves down by $x 0$, then $\angle \mathrm{DAB}=\angle \mathrm{ADC}=\varphi_{1}$ and $\angle \mathrm{GHF}=\angle \mathrm{HGE}=\varphi_{2}$. Under the action of the vibrated object, the length of diagonal spring and damper is, respectively, $\mathrm{AB}=\mathrm{CD}=l 1$ and $\mathrm{HF}=\mathrm{GE}=l 2$, with the following equations:

$$
\begin{aligned}
& l_{1}=\sqrt{l_{0}^{2} \cos ^{2} \varphi_{0}+\left(l_{0} \sin \varphi_{0}-x_{0}\right)^{2}}, \\
& l_{2}=\sqrt{l_{0}^{2} \cos ^{2} \varphi_{0}+\left(l_{0} \sin \varphi_{0}+x_{0}\right)^{2}} .
\end{aligned}
$$

In a static state, equilibrium equations of the vibrated object are as follows:

$$
\left\{\begin{array}{l}
4 K\left(l_{0}-l_{1}\right) \sin \varphi_{1}+4 K\left(l_{2}-l_{0}\right) \sin \varphi_{2}=M g \\
\sin \varphi_{1}=\frac{\left(l_{0} \sin \varphi_{0}-x_{0}\right)}{l_{1}}, \\
\sin \varphi_{2}=\frac{\left(l_{0} \sin \varphi_{0}+x_{0}\right)}{l_{2}} .
\end{array}\right.
$$

Set up a rectangular coordination system by taking the static equilibrium position as the origin of coordinates, with the positive direction being downward and displacement being $x$, then the length of diagonal spring and damper is, 


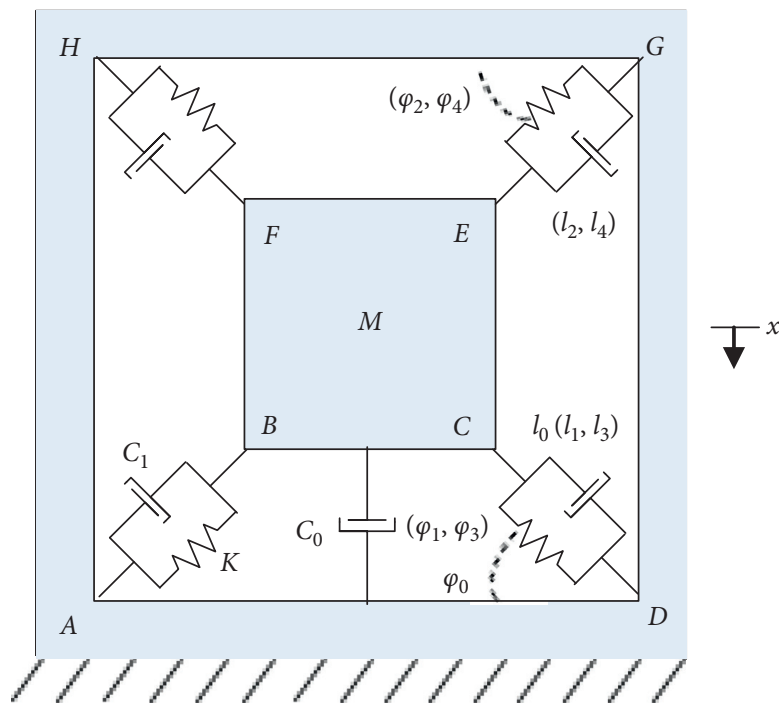

(a)

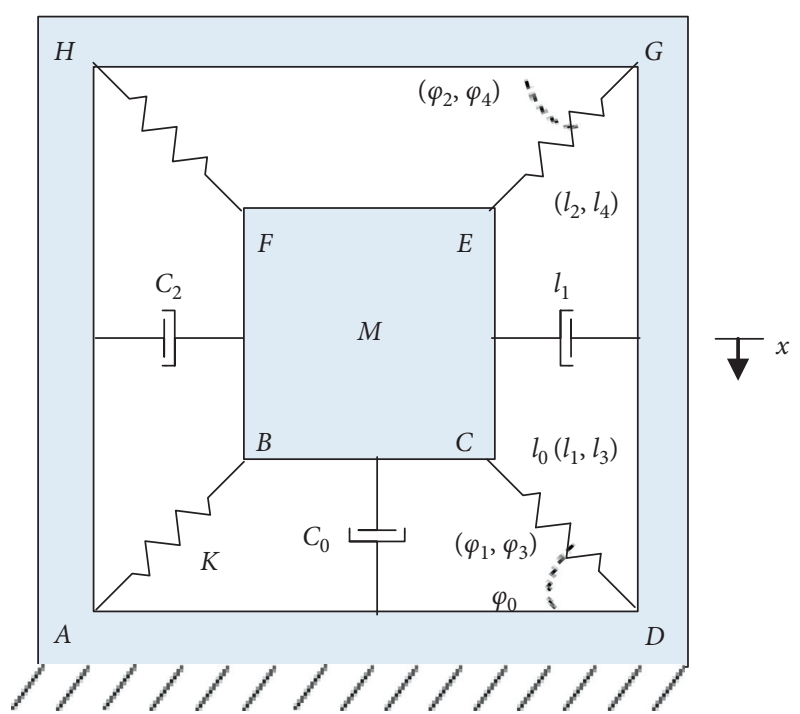

(b)

FIgURE 2: New types of SVRS with geometric nonlinear damping. (a) Type I. (b) Type II.

respectively: $\mathrm{AB}=\mathrm{CD}=13$ and $\mathrm{HF}=\mathrm{GE}=14$, with the following equations:

$$
\begin{aligned}
& l_{3}=\sqrt{l_{1}^{2} \cos ^{2} \varphi_{1}+\left(l_{1} \sin \varphi_{1}-x\right)^{2}}, \\
& l_{4}=\sqrt{l_{2}^{2} \cos ^{2} \varphi_{2}+\left(l_{2} \sin \varphi_{2}+x\right)^{2}} .
\end{aligned}
$$

$$
M \ddot{x}=M g-C_{0} \dot{x}-4 C_{1}\left(i_{0}-\dot{l}_{3}\right) \sin \varphi_{3}-4 C_{1}\left(i_{4}-\dot{l}_{0}\right) \sin \varphi_{4}-4 K\left(l_{0}-l_{3}\right) \sin \varphi_{3}-4 K\left(l_{4}-l_{0}\right) \sin \varphi_{4} \text {, }
$$

where $\sin \phi_{3}=\left(\left(l_{1} \sin \varphi_{1}-x\right) / l_{3}\right)$ and $\sin \varphi_{4}=\left(\left(l_{2} \sin \varphi_{2}\right.\right.$ $\left.+x) / l_{4}\right)$. Since $i_{0}=0$, the equation above can be converted to the following:

$$
M \ddot{x}=M g-C_{0} \dot{x}+4 C_{1} \dot{l}_{3} \sin \varphi_{3}-4 C_{1} i_{4} \sin \varphi_{4}-4 K\left(l_{0}-l_{3}\right) \sin \varphi_{3}-4 K\left(l_{4}-l_{0}\right) \sin \varphi_{4},
$$

where $\dot{l}_{3}=-\dot{x} \sin \varphi_{3}$ and $\dot{l}_{4}=\dot{x} \sin \varphi_{4} \cdot \sin \varphi_{3}, \sin \varphi_{4}, \dot{l}_{3}$ and $\dot{l}_{4}$ are substituted into formula (5), and notice that $\sin ^{2} \varphi_{3}=$ $1-\cos ^{2} \varphi_{3}=1-\left(l_{0} / l_{3}\right)^{2} \cos ^{2} \varphi_{0}$ $\sin ^{2} \varphi_{4}=1-\left(l_{0} / l_{4}\right)^{2} \cos ^{2} \varphi_{0}$; formula (5) can be finally converted to the following:

$$
\begin{aligned}
M \ddot{x}= & M g-C_{0} \dot{x}-4 C_{1} \dot{x}\left[1-\left(l_{0} / l_{3}\right)^{2} \cos ^{2} \varphi_{0}\right]-4 C_{1} \dot{x}\left[1-\left(l_{0} / l_{4}\right)^{2} \cos ^{2} \varphi_{0}\right] \\
& -4 K\left(\left(l_{0} / l_{3}\right)-1\right)\left(l_{0} \sin \varphi_{0}-x_{0}-x\right)-4 K\left(1-\left(l_{0} / l_{4}\right)\right)\left(l_{0} \sin \varphi_{0}+x_{0}+x\right) .
\end{aligned}
$$


Under the premise of omitting high-order term and ignoring small quantity, conduct Taylor series expansion of $10 / 13$ and $10 / 14$ to obtain the following equations:

$$
\begin{aligned}
\frac{l_{0}}{l_{3}} & =\frac{1}{\sqrt{1-\left(\left(2\left(x_{0}+x\right) l_{0} \sin \varphi_{0}-\left(x_{0}+x\right)^{2}\right) / l_{0}^{2}\right)}}, \\
& =1+\frac{x_{0}+x}{l_{0}} \sin \varphi_{0}-\frac{1}{2 l_{0}^{2}}\left(x_{0}+x\right)^{2}+\frac{3}{2 l_{0}^{2}} \sin ^{2} \varphi_{0}\left(x_{0}+x\right)^{2}-\frac{3}{2 l_{0}^{3}} \sin \varphi_{0}\left(x_{0}+x\right)^{3}+\frac{5}{2 l_{0}^{3}} \sin ^{3} \varphi_{0}\left(x_{0}+x\right)^{3}, \\
\frac{l_{0}}{l_{4}} & =1-\frac{x_{0}+x}{l_{0}} \sin \varphi_{0}-\frac{1}{2 l_{0}^{2}}\left(x_{0}+x\right)^{2}+\frac{3}{2 l_{0}^{2}} \sin ^{2} \varphi_{0}\left(x_{0}+x\right)^{2}+\frac{3}{2 l_{0}^{3}} \sin \varphi_{0}\left(x_{0}+x\right)^{3}-\frac{5}{2 l_{0}^{3}} \sin ^{3} \varphi_{0}\left(x_{0}+x\right)^{3} .
\end{aligned}
$$

Substitute the computed results above into the right half part of formula (6) and omit the high-order term (threeorder above) to obtain the following equation:

$$
\begin{aligned}
M \ddot{x}= & M g-C_{0} \dot{x}-8 C_{1}\left[\sin ^{2} \varphi_{0}+\frac{1}{l_{0}^{2}}\left(\cos ^{2} \varphi_{0}-4 \cos ^{2} \varphi_{0} \sin ^{2} \varphi_{0}\right)\left(x_{0}+x\right)^{2}\right] \dot{x} \\
& -8 K\left[\sin ^{2} \varphi_{0}\left(x_{0}+x\right)+\frac{1}{2 l_{0}^{2}}\left(1-6 \sin ^{2} \varphi_{0}+5 \sin ^{4} \varphi_{0}\right)\left(x_{0}+x\right)^{3}\right] .
\end{aligned}
$$

According to formula (2),

$$
M g=4 K\left(\frac{l_{0}}{l_{1}}-1\right)\left(l_{0} \sin \varphi_{0}-x_{0}\right)+4 K\left(1-\frac{l_{0}}{l_{2}}\right)\left(l_{0} \sin \varphi_{0}+x_{0}\right) \text {. }
$$

Conduct Taylor series expansion of $10 / 11$ and 10/12 in reference to the expansion method of $10 / 13$ and $10 / 14$ and substitute the results obtained into the right half part of formula (9) to obtain the following equation:

$$
M g=8 K\left[\sin ^{2} \varphi_{0} x_{0}+\frac{1}{2 l_{0}^{2}}\left(1-6 \sin ^{2} \varphi_{0}+5 \sin ^{4} \varphi_{0}\right) x_{0}^{3}\right]
$$

Substitute formula (10) into formula (8) and assume that $\mathrm{x} 0$ is a small quantity, then

$$
M \ddot{x}+C_{0} \dot{x}+8 C_{1}\left[\sin ^{2} \varphi_{0}+\frac{1}{l_{0}^{2}}\left(\cos ^{2} \varphi_{0}-4 \cos ^{2} \varphi_{0} \sin ^{2} \phi_{0}\right) x^{2}\right] \dot{x}+8 K\left[\sin ^{2} \varphi_{0} x+\frac{1}{2 l_{0}^{2}}\left(1-6 \sin ^{2} \varphi_{0}+5 \sin ^{4} \varphi_{0}\right) x^{3}\right]=0
$$

Assuming that $a_{0}=\sin ^{2} \phi_{0}, b_{0}=\left(\left(1-6 \sin ^{2} \varphi_{0}+5 \sin ^{4}\right.\right.$ $\left.\varphi_{0}\right) / 2$ ) and $d_{0}=\cos ^{2} \varphi_{0}-4 \cos ^{2} \varphi_{0} \sin ^{2} \varphi_{0}$, formula (11) can be shown as follows:

$$
M \ddot{x}+C_{0} \dot{x}+8 C_{1}\left(a_{0}+\frac{d_{0}}{l_{0}^{2}} x^{2}\right) \dot{x}+8 K\left(a_{0} x+\frac{b_{0}}{l_{0}^{2}} x^{3}\right)=0 .
$$

\subsection{Establishment of Motion Differential Equation for Type II.} It can be known from Figure 2 that Type II has the same spring layout as Type I, and both of them have diagonal spring, so they have the same resilience in a vertical direction, which is not deduced again here, with the equation as below:

$$
F_{S}=8 K\left(a_{0} x+\frac{b_{0}}{l_{0}^{2}} x^{3}\right) .
$$

However, Type II has a different damper layout from Type I, so the damping force in the vertical direction will be different, and the formula of vertical damping force of Type II is deduced as below. In Type II, the horizontal damping is $C 2$, the length is 11 , other parameters are the same as those of Type I, and the initial position refers to a horizontal position. After the vibrated object moves down by $x$, the length of horizontal damping $C 2$ is as follows: 


$$
z=\sqrt{x^{2}+l_{1}^{2}}
$$

Correspondingly, the damping force generated by each horizontal damping $C 2$ is as follows:

$$
F_{D i}=C_{2} z^{\prime}
$$

Take the derivative of $z$, then

$$
z^{\prime}=\frac{x \dot{x}}{\sqrt{x^{2}+l_{1}^{2}}}
$$

Vertical damping force generated by each horizontal damping $C 2$ can be expressed as follows:

$$
F_{V i}=F_{D i} \sin \theta
$$

where $\theta$ indicates the included angle between $C 2$ and horizontal direction after the vibrated object moves down by $x$. Geometrically, $\sin \theta$ can be expressed as follows:

$$
\sin \theta=\frac{x}{\sqrt{x^{2}+l_{1}^{2}}}
$$

Refer to the formula of $\sin \theta$ and substitute $z^{\prime}$ into FVi, then FVi can be expressed as follows:

$$
F_{V i}=C_{2} \dot{x} \sin ^{2} \theta .
$$

Conduct Taylor series expansion of $\sin \theta$ to obtain the following formula:

$$
\sin \theta \approx \theta-\frac{\theta^{3}}{3 !}+\cdots .
$$

Assuming that the vibrated object vibrates in a small range, namely, $\theta$ is small, then

$$
\theta \approx \frac{x}{l_{1}} \text {. }
$$

Take the first-order term of $\sin \theta$ Taylor series into the formula of FVi, and FVi can be changed as follows:

$$
F_{V i}=\frac{C_{2}}{l_{1}^{2}} \dot{x} x^{2} .
$$

When the two horizontal dampings act together, total vertical damping force can be expressed as follows:

$$
F_{V}=2 \frac{C_{2}}{l_{1}^{2}} \dot{x} x^{2}
$$

The formula above refers to the vertical damping force of two horizontal dampings. Through the derivation above, the motion differential equation of Type II can be expressed as follows:

$$
M \ddot{x}+C_{0} \dot{x}+\frac{2 C_{2}}{l_{1}^{2}} x^{2} \dot{x}+8 K\left(a_{0} x+\frac{b_{0}}{l_{0}^{2}} x^{3}\right)=0
$$

2.3. Comparison of Motion Differential Equations. According to the literature [18], the motion differential equation of GSVRS is shown as follows:

$$
M \ddot{x}+C_{0} \dot{x}+8 K\left(a_{0} x+\frac{b_{0}}{l_{0}^{2}} x^{3}\right)=0 .
$$

According to formula (12), formula (24), and formula (25), in comparison with the motion differential equation of GSVRS, there is nonlinear damping force $8 C_{1}\left(\left(a_{0}+\right.\right.$ $\left.\left.d_{0} x^{2}\right) / l_{0}^{2}\right) \dot{x}$ and $\left(2 C_{2} x^{2} \dot{x} / l_{1}^{2}\right)$ added in the motion differential equation of Type I and Type II, respectively. Since linear damping is adopted in this paper, the nonlinear damping force generated mainly results from spatial geometrical factors. Thus, the damping force obtained in this paper can be deemed as a geometric nonlinear damping force.

It can be known from $8 C_{1}\left(\left(a_{0}+d_{0} x^{2}\right) / l_{0}^{2}\right) \dot{x}$ that geometric nonlinear damping force of Type I comprises not only linear part but also a nonlinear part which is related to the square displacement. According to $\left(2 C_{2} x^{2} \dot{x} / l_{1}^{2}\right)$, the geometric nonlinear damping force of Type II comprises nonlinear part only, which is related to the square displacement. Compared with classic cubic nonlinear force, the geometric nonlinear damping forces of Type I and Type II are more complex, which have no obvious characteristics of becoming soft or hard [19]; instead, the damping forces will form different curved surfaces together with the displacement and velocity based on different system parameters, which are unpredictable, thus causing much inconvenience in subsequent analysis.

The variation rule of the geometric nonlinear damping forces is studied below. It can be known from the expression of geometric nonlinear damping force in Type I that, according to different suspension angles $\varphi 0, \mathrm{~d} 0$ can be either positive or negative. Therefore, this paper provides two sets of parameters to calculate the curved surface of geometric nonlinear damping force $8 C_{1}\left(\left(a_{0}+d_{0} x^{2}\right) / l_{0}^{2}\right) \dot{x}$ : (I) $\varphi_{0}=\pi / 4$, $C 1=1000 \mathrm{~N} \mathrm{~s} / \mathrm{m}$, and $l 0=0.1 \mathrm{~m}$; (II) $\varphi 0=\pi / 7, C 1=1000 \mathrm{~N} \mathrm{~s} /$ $\mathrm{m}$, and $l 0=0.1 \mathrm{~m}$. For Type II, only one set of parameters is provided to calculate the curved surface of geometric nonlinear damping force: $C 2=1000 \mathrm{~N} \mathrm{~s} / \mathrm{m}$, and $l 1=0.1 \mathrm{~m}$. Assuming the variation range of velocity is $(0-4 \mathrm{~m} / \mathrm{s})$ and that of displacement is $(0-0.1 \mathrm{~m})$, the curved surface of geometric nonlinear damping force in Type I and Type II is, respectively, shown in Figures 3 and 4 .

According to the formula of geometric nonlinear damping force in Type I, when the displacement is $0 \mathrm{~m}$, the damping force related to square displacement is $0 \mathrm{~N}$, and the whole geometric nonlinear damping force comprises linear part only regardless of $\mathrm{d} 0$ (positive or negative). However, with the increase of displacement, the damping force related to square displacement has an increasing proportion in the whole geometric nonlinear damping force. When $\mathrm{d} 0$ is positive, namely, the suspension angle $\varphi 0$ is small, the damping force related to square displacement will increase the whole damping force; when $\mathrm{d} 0$ is negative, namely, the suspension angle $\varphi 0$ is large, the damping force related to square displacement will reduce the whole damping force. When the displacement is small, the damping force in Figure 3(a) is greater than that in Figure 3(b). With the increase of displacement, the damping force in Figure 3(a) becomes smaller than that in 


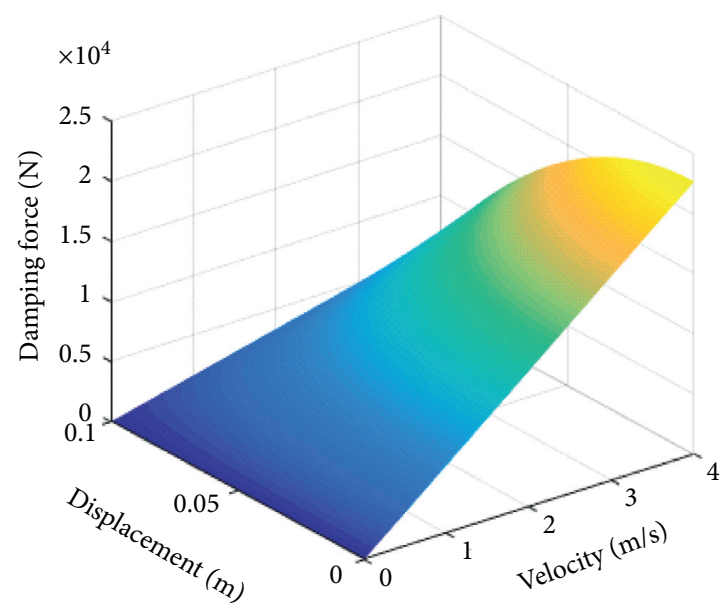

(a)

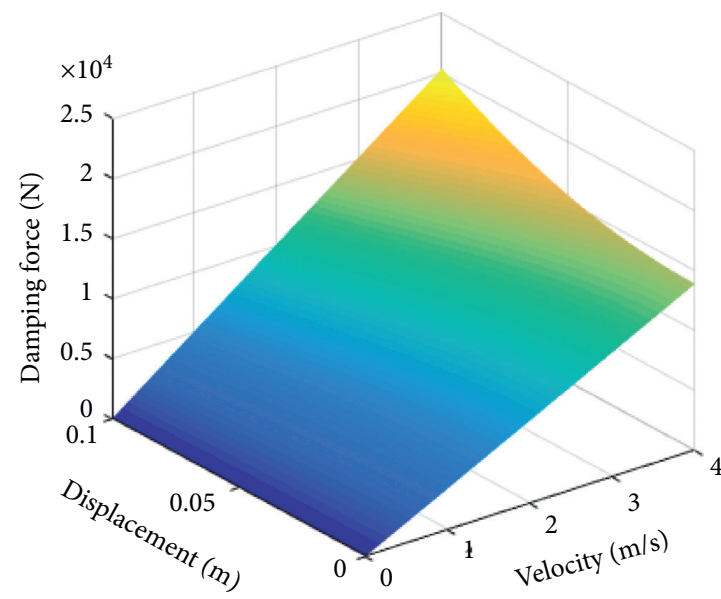

(b)

FIGURE 3: Geometric nonlinear damping force in Type I. (a) Geometric nonlinear damping force corresponding to parameter (I). (b) Geometric nonlinear damping force corresponding to parameter II.

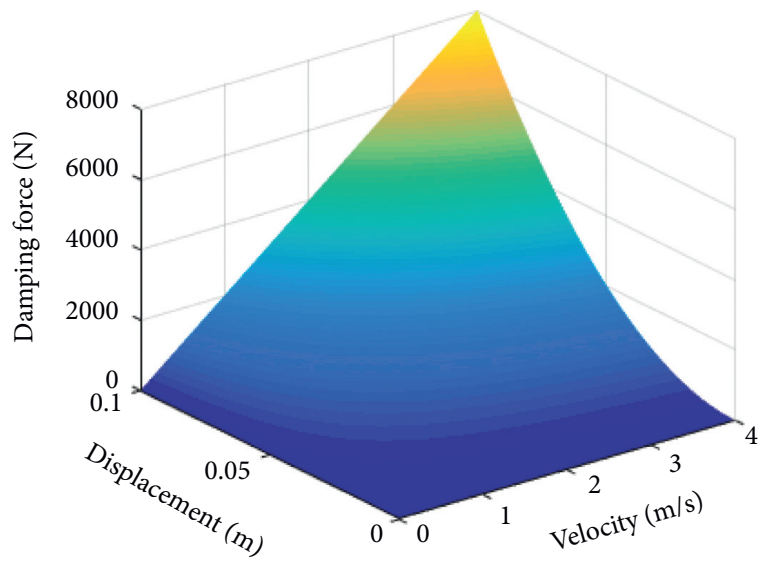

Figure 4: Geometric nonlinear damping force in Type II.

Figure 3(b). It indicates that large $\varphi 0$ is good for the vibration with small amplitude and small $\varphi 0$ is the opposite. According to the formula of $\mathrm{d} 0$, only when $\varphi 0$ is smaller than $\pi / 6$ can $\mathrm{d} 0$ be positive.
The geometric nonlinear damping force in Type II has a similar variation rule to that in Type $\mathrm{I}$, of which $\mathrm{d} 0$ is positive, both of them rise with the increase of displacement and velocity; however, since the damping force in Type II has no linear part, it varies dramatically with the change of displacement and velocity; and when the displacement is $0 \mathrm{~m}$, the whole damping force will be $0 \mathrm{~N}$. In addition, due to lack of linear damping force, the whole geometric nonlinear damping force in Type II is smaller than that in Type I of which $\mathrm{d} 0$ is positive.

\section{Comparative Study}

3.1. Comparative Study under Impact Excitation. In order to further study the difference among Type I, Type II, and GSVRS, we will conduct a comparative study on the attenuation effects of the three types of SVRS on impact excitation. To get close to engineering practice, in consideration of the base motion and assuming that the vibration displacement of outer frame ADGH is $q$, formula (12), formula (24), and formula (25) can be changed as follows:

$$
\begin{aligned}
M \ddot{x}+C_{0}(\dot{x}+\dot{q})+8 C_{1}\left[a_{0}+\frac{d_{0}}{l_{0}^{2}}(x+q)^{2}\right](\dot{x}+\dot{q})+8 K\left[a_{0}(x+q)+\frac{b_{0}}{l_{0}^{2}}(x+q)^{3}\right] & =0, \\
M \ddot{x}+C_{0}(\dot{x}+\dot{q})+\frac{2 C_{2}}{l_{1}^{2}}(x+q)^{2}(\dot{x}+\dot{q})+8 K\left[a_{0}(x+q)+\frac{b_{0}}{l_{0}^{2}}(x+q)^{3}\right] & =0, \\
M \ddot{x}+C_{0}(\dot{x}+\dot{q})+8 K\left[a_{0}(x+q)+\frac{b_{0}}{l_{0}^{2}}(x+q)^{3}\right] & =0 .
\end{aligned}
$$


Assuming $y=(x+q) / 10$, conduct dimensionless processing of formula (26), formula (27), and formula (28) to obtain the following equations:

$$
\frac{\mathrm{d}^{2} y}{\mathrm{~d} \tau^{2}}+2 \xi_{0} \frac{\mathrm{d} y}{\mathrm{~d} \tau}+2 \xi_{1}\left(a_{0}+\mathrm{d}_{0} y^{2}\right) \frac{\mathrm{d} y}{\mathrm{~d} \tau}+\left(a_{0} y+b_{0} y^{3}\right)=\beta \ddot{q},
$$

$$
\frac{\mathrm{d}^{2} y}{\mathrm{~d} \tau^{2}}+2 \xi_{0} \frac{\mathrm{d} y}{\mathrm{~d} \tau}+2 \xi_{2} L^{2} y^{2} \frac{\mathrm{d} y}{\mathrm{~d} \tau}+\left(a_{0} y+b_{0} y^{3}\right)=\beta \ddot{q}
$$

$\frac{\mathrm{d}^{2} y}{\mathrm{~d} \tau^{2}}+2 \xi_{0} \frac{\mathrm{d} y}{\mathrm{~d} \tau}+\left(a_{0} y+b_{0} y^{3}\right)=\beta \ddot{q}$,

where $\omega_{0}=\sqrt{(8 K / M)}$ is the frequency parameter of the system, $\xi_{0}=\left(C_{0} /(2 \sqrt{8 K M})\right)$ is the vertical damping ratio of the system, $\xi_{1}=\left(8 C_{1} /(2 \sqrt{8 K M})\right)$ is the diagonal damping ratio of Type I, $\xi_{2}=\left(2 C_{2} /(2 \sqrt{8 K M})\right)$ is the lateral damping ratio of Type II, $T=\left(1 / \omega_{0}\right)$ is the cycle parameter of the system, $\tau=(t / T)=\omega_{0} t$ is the dimensionless time, $\tau_{0}=\left(t_{0} / T\right)$ is the dimensionless impact excitation time, $\beta=$ $\left(T^{2} / l_{0}\right)$ is the feature parameter of the system, and $L=10 /$ $11=1 / \cos \varphi 0$ is the damping length ratio. The impact excitation is represented by a half-sine wave of acceleration, which can be expressed as follows:

$$
\ddot{q}= \begin{cases}A \sin (\omega t), & 0 \leq t \leq t_{0}, \\ 0, & t>t_{0} .\end{cases}
$$

Assuming that the frequency ratio of the system $s=\left(\omega / \omega_{0}\right)$,

$$
\beta \ddot{q}= \begin{cases}\beta A \sin (s \tau), & 0 \leq \tau \leq \tau_{0} \\ 0, & t>\tau_{0} .\end{cases}
$$

This paper refers to the comparative study on the vibration isolation performance of three types of SVRS by taking the dimensionless acceleration of a vibrated object as the measurement index, with the dimensionless acceleration of vibrated object being expressed as follows:

$$
\begin{aligned}
x^{\prime \prime} & =\frac{\mathrm{d}^{2}\left(x / l_{0}\right)}{\mathrm{d} \tau^{2}}=\frac{\mathrm{d}^{2} y}{\mathrm{~d} \tau^{2}}-\frac{\mathrm{d}^{2} q}{l_{0} \mathrm{~d} \tau^{2}}, \\
& =\frac{\mathrm{d}^{2} y}{\mathrm{~d} \tau^{2}}-\frac{T^{2} \mathrm{~d}^{2} q}{l_{0} \mathrm{~d} t^{2}}=\frac{\mathrm{d}^{2} y}{\mathrm{~d} \tau^{2}}-\beta \ddot{q} .
\end{aligned}
$$

Before calculation, the required parameters shall be provided firstly. By observing the formulas of $\mathrm{b} 0$ and $\mathrm{d} 0, \mathrm{~b} 0$ and $\mathrm{d} 0$ can be either positive or negative based on different suspension angles $\varphi 0$. Through the calculation in Section 2, only when $\varphi 0<\pi / 6=0.5328$, can $\mathrm{d} 0$ be positive, while for b0, only when $\varphi 0<0.4636$, can b0 be positive; thus three situations may occur to b0 and d0: (1) when $\varphi 0<0.4636$, both $\mathrm{b} 0$ and $\mathrm{d} 0$ are positive; (2) when $0.4636<\varphi 0<\pi / 6$, b0 is negative and $\mathrm{d} 0$ is positive; and (3) when $\varphi 0>\pi / 6$, both $\mathrm{b} 0$ and $\mathrm{d} 0$ are negative. Therefore, this paper provides three sets of parameters: (I) $\varphi 0=\pi / 7$, $a 0=0.1883, b 0=0.0238, d 0=0.2005, \omega 0=58.8269, \zeta 0=0.1$, $\zeta 1=0.3, \zeta 2=0.5, \quad T=0.017, \quad \beta=0.0029, \quad L=1.1099, \quad A=49$, $s=5.3404$, and $\tau 0=0.5883$; (II) $\varphi 0=0.5, a 0=0.2298, b 0=-$
$0.0575, d 0=0.0621, \omega 0=53.4652, \zeta 0=0.1, \zeta 1=0.3, \zeta 2=0.5$, $T=0.0187, \quad \beta=0.0035, \quad L=1.1395, \quad A=49, \quad s=5.876$, and $\tau 0=0.5347$; and (III) $\varphi 0=\pi / 4, a 0=0.5, b 0=-0.375, d 0=-0.5$, $\omega 0=36.4568, \zeta 0=0.1, \zeta 1=0.3, \zeta 2=0.5, T=0.0274, \beta=0.0075$, $L=1.4142, A=49, s=8.6173$, and $\tau 0=0.3646$. This paper adopts the improved partitioning and dimensional increment precise integration method [20] to solve the dimensionless equations (29), (30), and (31). Through calculation, the dimensionless acceleration of vibrated object corresponding to parameter I, parameter II, and parameter III is shown in Figures 5-7, respectively.

It can be found from Figures 5-7 that, under the premise of $\zeta 2>\zeta 1>\zeta 0$, the dimensionless acceleration response curve of Type II almost totally coincides with that of GSVRS; from the partial view, the acceleration response curve of Type II is slightly smaller than that of GSVRS at the peak, which can be ignored since the difference is tiny. Thus, it can be concluded that the added lateral damping is almost unhelpful to improve the vibration isolation performance of SVRS under the effect of vertical impact. Conversely, the difference between the response curve of Type I and that of GSVRS is greatly obvious. The dimensionless acceleration response curves of the two systems are basically the same at the first peak; however, the vibration isolation performance of Type I is superior to that of GSVRS at the second peak and following peaks, especially in the situation of parameter III, Type I is obviously superior to GSVRS no matter in peak value or peak number.

The reason that Type I has the vibration isolation performance superior to GSVRS, while Type II has the vibration isolation performance substantially the same as GSVRS is as follows: diagonal damping generates both geometric nonlinear damping force related to the square displacement and linear damping force of Type I, while lateral damping only generates geometric nonlinear damping force related to the square displacement of Type II. In a vibration system, displacement is often a small physical parameter, and it becomes much smaller after being squared, so does the product of damping force, which causes that the geometric nonlinear damping force related to the square displacement is small, as shown in Figures 3 and 4. Therefore, in comparison with linear damping force, the geometric nonlinear damping force related to the square displacement against impact is limited, so that Type I has the vibration isolation performance superior to GSVRS, while Type II has the vibration isolation performance substantially the same as GSVRS.

Furthermore, it can be found through comparison among Figures 5-7 that, no matter SVRS has geometric nonlinear damping or not, the amplitude of dimensionless acceleration response curve rises correspondingly with the increase of suspension angle $\varphi 0$, that is, the trend of parameter III being greater than parameter II and parameter II being greater than parameter I exist in the aspect of amplitude. It indicates that during the design of SVRS, the suspension angle $\varphi 0$ should be as small as possible to ensure that both $b 0$ and $d 0$ are positive. Note: the suspension angle $\varphi 0$ should meet the practical requirement. 


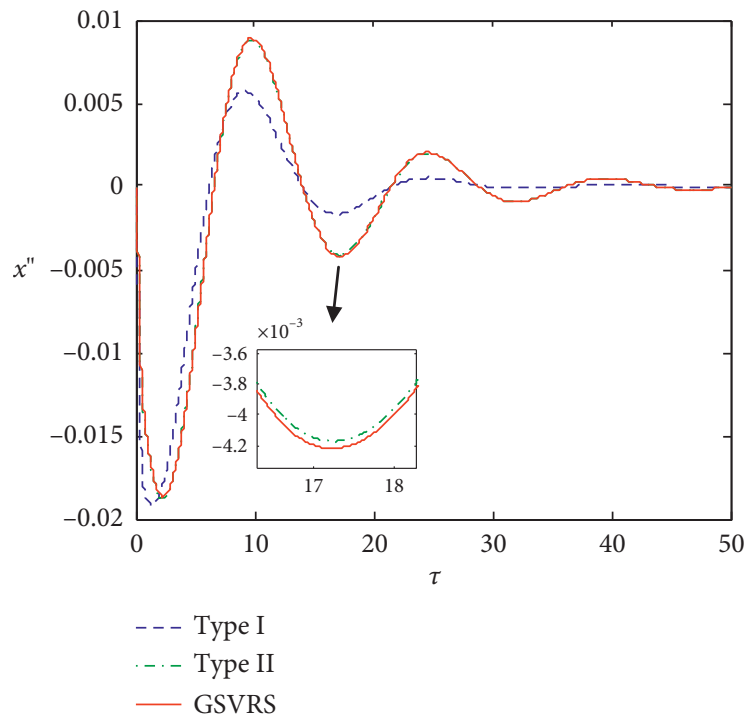

FIGURE 5: Dimensionless acceleration response of parameter I.

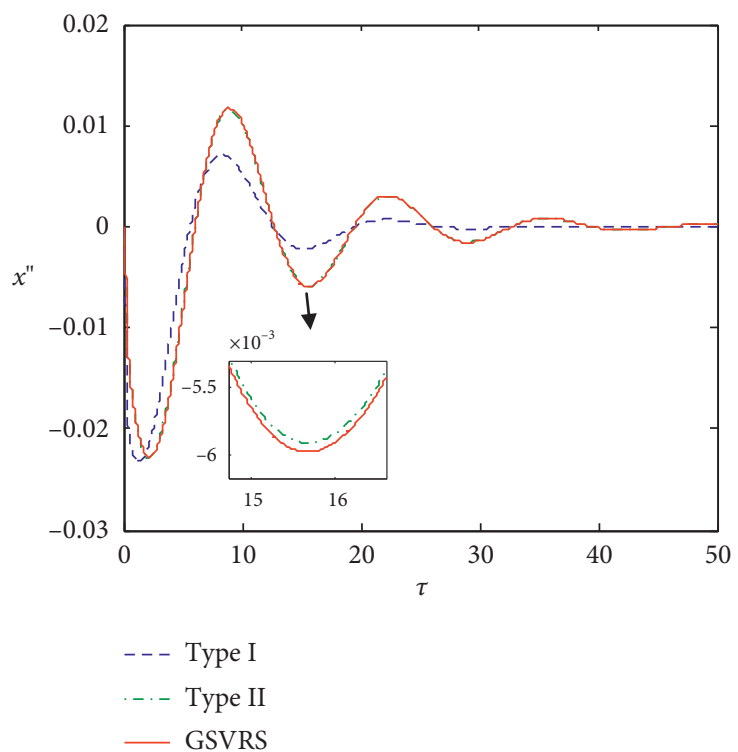

FIGURE 6: Dimensionless acceleration response of parameter II.

3.2. Comparative Study under Random Excitation. Conduct a comparative study on the vibration isolation performance of Type I, Type II, and GSVRS under random excitation in reference to the study method of Section 3.1. Dimensionless excitation $\ddot{q}$ is represented by white Gaussian noise in this section, with the excitation time $\tau 0=5882.7$, and the sampling number being 1000 per unit time; and the dimensionless waveform and probability density distribution are shown in Figures 8 and 9.

The improved partitioning and dimensional increment precise integration method is adopted to solve equations (29), (30), and (31) by taking the probability density distribution of dimensionless acceleration (as shown in equation (34)) of the vibrated object as the evaluation index, so as to obtain the comparison among the probability density distributions corresponding to parameter I to parameter III in Section 3.1, as shown in Figures 10-12.

It can be known from Figures 10-12 that, under three different parameter conditions, the probability density distribution of Type II is totally the same as that of GSVRS, which indicates that the adding of lateral damping is unhelpful for the attenuation of random excitation. The probability density distribution values of Type I around zero are greater than those of both Type II and GSVRS, which indicates that the adding of diagonal damping is very helpful for the attenuation of random excitation. Through the attenuation of Type I, the acceleration of the vibrated object is more concentrated at zero, and the vibration isolation performance is superior to that of Type II and GSVRS, the reason of which has been discussed in Section 3.1, thus is not 


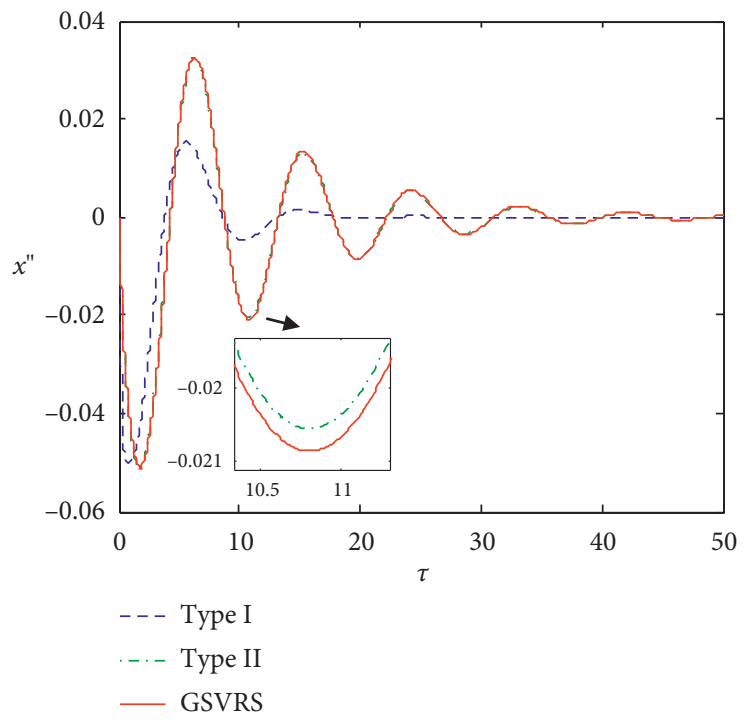

FIgURE 7: Dimensionless acceleration response of parameter III.

repeated here. Furthermore, it can be found through the comparison among Figures 10-12 that, with the increase of suspension angle $\varphi 0$, the concentration of vibrated object acceleration at zero decreases correspondingly, which indicates that during the design of SVRS, the suspension angle $\varphi 0$ should be as small as possible, and such conclusion is consistent to the study result of Section 3.1.

The comparative study of this section shows that the adding of diagonal damping improves the vibration isolation performance of SVRS under impact excitation and random excitation, while that of lateral damping has no such effect. Comprehensively, Type I is superior to Type II. Therefore, the optimization analysis of Type I will be carried out in Section 4 .

\section{Optimization Analysis}

Through a comparative study on the vibration isolation performance of Type I, Type II, and GSVRS in Section 3, it can be concluded that diagonal damping is better than lateral damping at improving the vibration isolation performance of SVRS, so the vibration isolation performance of Type I is better than that of Type II and GSVRS; however, the influence rule of Type I parameters on vibration isolation performance has not been known yet. Therefore, this section refers to the study on the influence rule of Type I parameters on vibration isolation performance by using the ergodic optimization method and seeks the optimum parameters of Type I on this basis.

The ergodic optimization method [21, 22] is as follows: under the premise of meeting the calculation accuracy, conduct uniform sampling of each parameter range and then calculate the objective function value under each parameter combination, respectively, so as to select the optimal value and parameter setting and observe the variation rule of objective function value with the variation of system parameters. Prior to ergodic optimization, the objective function shall be given first. It can be known through the analysis in Section 3 that the study results under impact

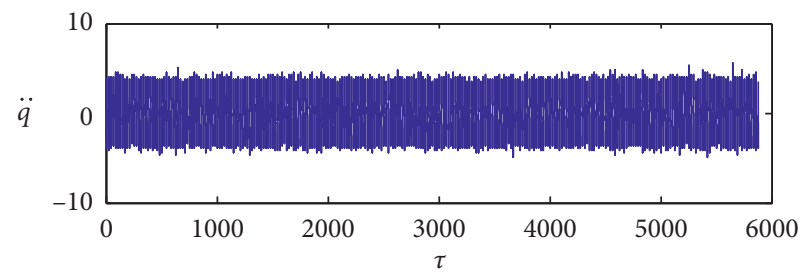

Figure 8: Dimensionless waveform of white Gaussian noise.

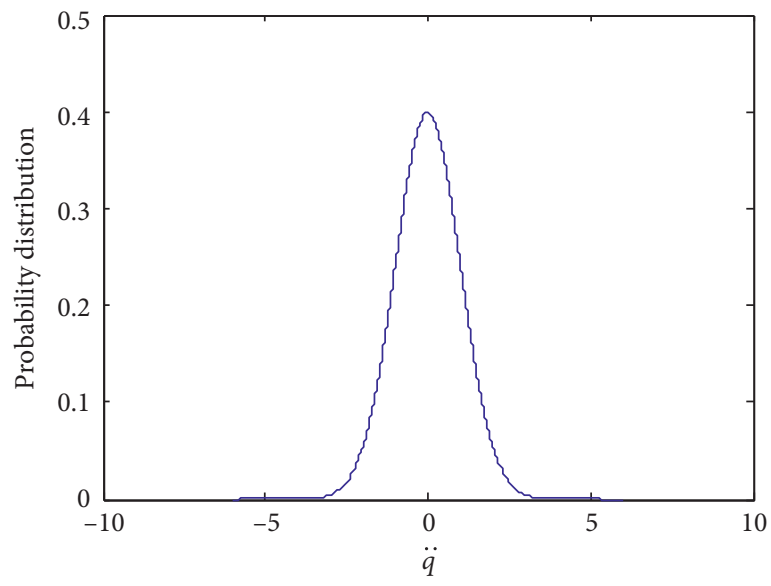

Figure 9: Probability density distribution.

excitation and random excitation are basically the same; however, since random excitation is more common, the objective function refers to the root-mean-square (RMS) value of vibrated object acceleration under random excitation, with the calculation formula shown as follows:

$$
x_{\mathrm{RMS}}^{\prime \prime}=\sqrt{\frac{\sum_{i=1}^{N}\left(x_{i}^{\prime \prime}\right)^{2}}{N}},
$$




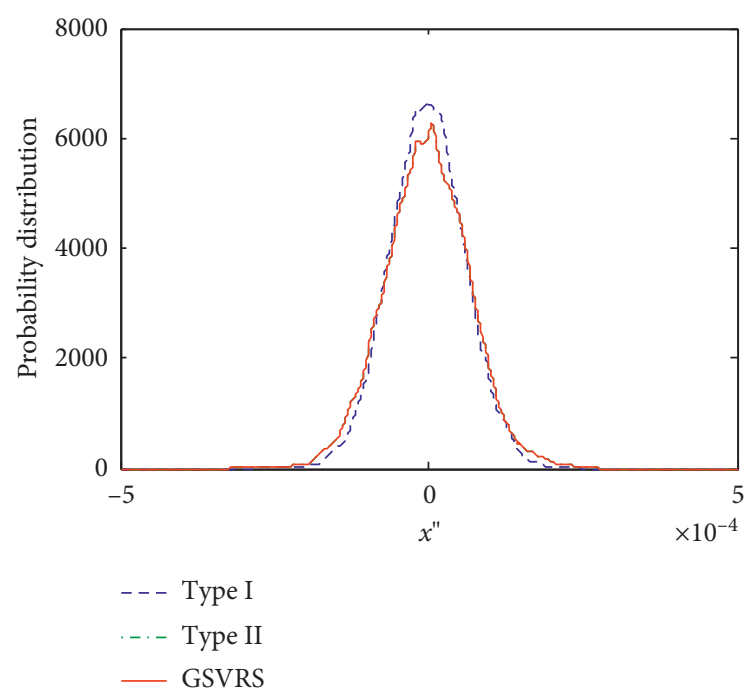

FIgURE 10: Probability density distribution corresponding to parameter I.

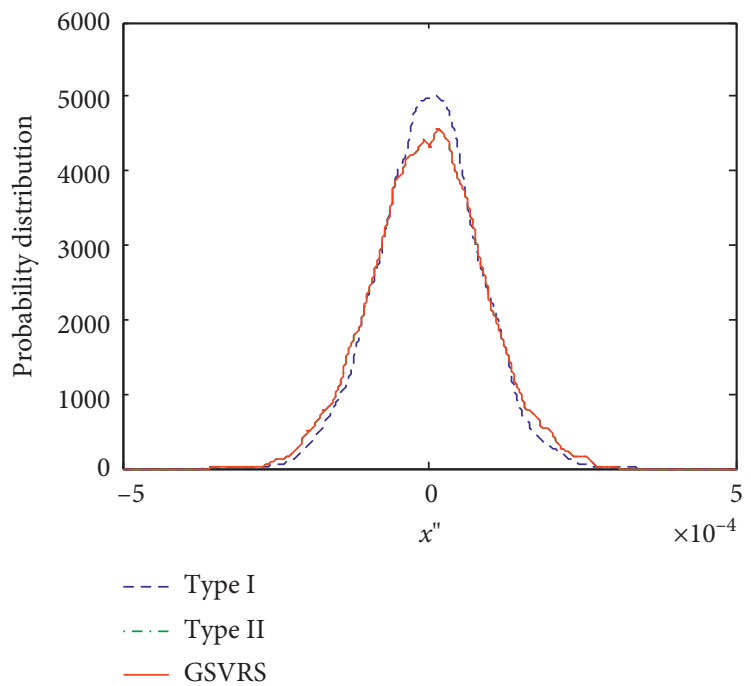

FIgURE 11: Probability density distribution corresponding to parameter II.

where $x^{\prime \prime}$ is the dimensionless acceleration of the vibrated object, with the calculation method shown in formula (34). After the objective function is given, it is required to provide the variation range of parameters. It can be observed from formula (29) that the parameters influencing vibration isolation performance of Type I mainly refer to $\zeta 0, \zeta 1, \mathrm{a} 0, \mathrm{~b} 0$, $\mathrm{d} 0$, and $\beta$, among which $\mathrm{a} 0, \mathrm{~b} 0$, and $\mathrm{d} 0$ are determined upon the suspension angle $\varphi 0$, so the parameters above can be finally simplified to four parameters $(\zeta 0, \zeta 1, \varphi 0$, and $\beta)$. Assuming that the system parameters conform to uniform variation [23], with parameter II in Section 3 as the center, the variation range of the four parameters above is shown in Table 1 when the positive/negative variation reaches $80 \%$.

Divide the data in Table 1 into 20 equal shares, then conduct ergodic optimization, and adopt the improved partitioning and dimensional increment precise integration method to calculate the values of objective function under random excitation $\ddot{q}$ as shown in Figures 8 and 9 , so as to obtain the distribution of objective function values, as shown in Figures 13-16.

According to Figures 13 and 14, with the increase of $\zeta 0$ and $\zeta 1$, the variation range of RMS value of vibrated object acceleration becomes small, and the maximum value decreases, which indicates that increase of $\zeta 0$ and $\zeta 1$ is helpful to improve the vibration isolation performance of Type I since the increase of $\zeta 0$ and $\zeta 1$ can increase the damping force of Type I and the increase of damping force can improve the vibration isolation performance of Type I. According to Figures 15 and 16, with the increase of $\varphi 0$ and $\beta$, the variation range of RMS value of vibrated object acceleration becomes large, and the maximum value rises correspondingly, which indicates that decrease of $\varphi 0$ and $\beta$ can reduce the RMS value of vibrated object acceleration; the phenomenon that the decrease of $\varphi 0$ can reduce the RMS value is consistent to the study result in Section 3, while the reason that the decrease of $\beta$ can reduce the RMS value is as 


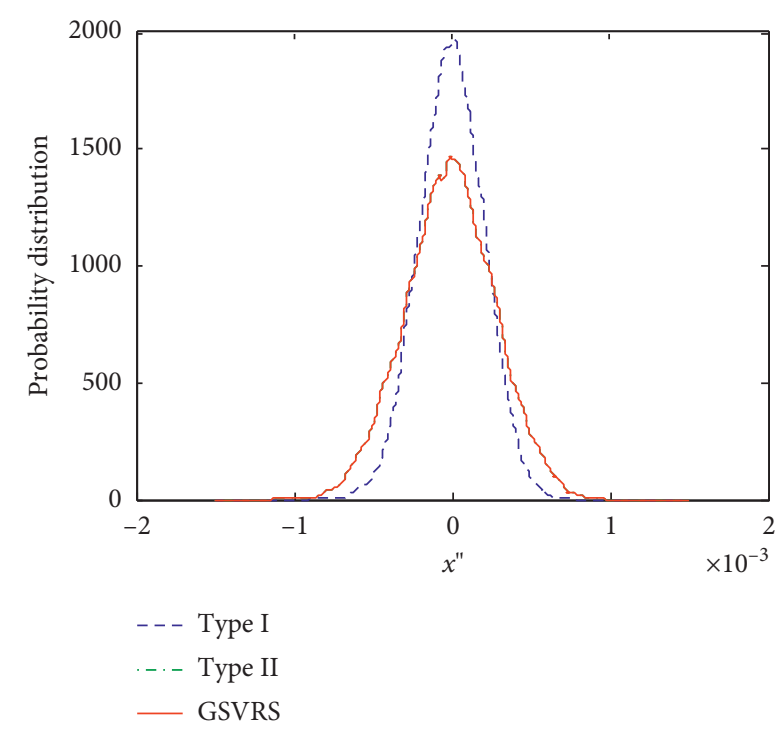

Figure 12: Probability density distribution corresponding to parameter III.

TABLE 1: Variation range of parameters.

\begin{tabular}{lcc}
\hline Parameters & Minimum & Maximum \\
\hline$\zeta_{0}$ & 0.02 & 0.18 \\
$\zeta_{1}$ & 0.06 & 0.54 \\
$\varphi_{0}$ & 0.1 & 0.9 \\
$\beta$ & 0.0007 & 0.0063 \\
\hline
\end{tabular}

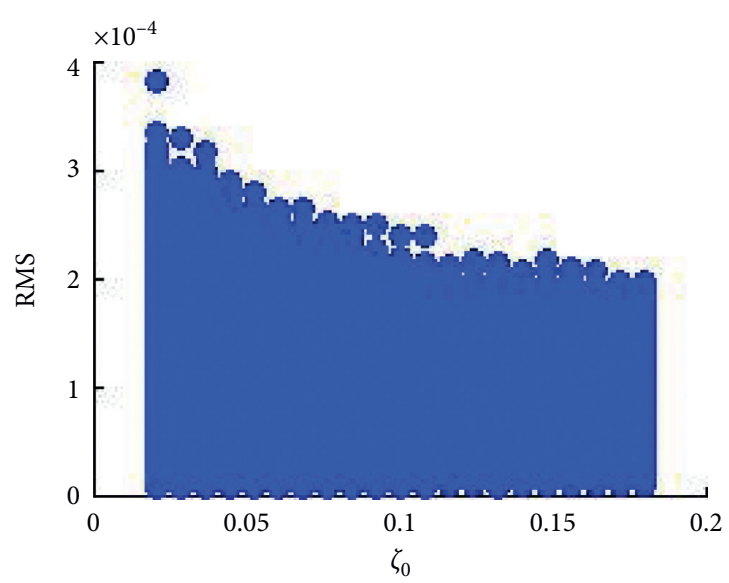

FIGURE 13: The distribution of calculation results corresponding to $\zeta 0$.

follows: multiplicative relationship exists between $\beta$ and excitation, and decrease of $\beta$ can reduce the whole excitation, thus reducing the RMS value of vibrated object acceleration. Comprehensively, $\zeta 0$ and $\zeta 1$ should be as big as possible and $\varphi 0$ and $\beta$ should be as small as possible during the design of Type I.

Furthermore, the optimal parameters of Type I can be obtained through calculation. Within the variation range of parameters, the minimum RMS value of vibrated object acceleration is $5.9102 \times 10^{-6}$. The corresponding dimensionless

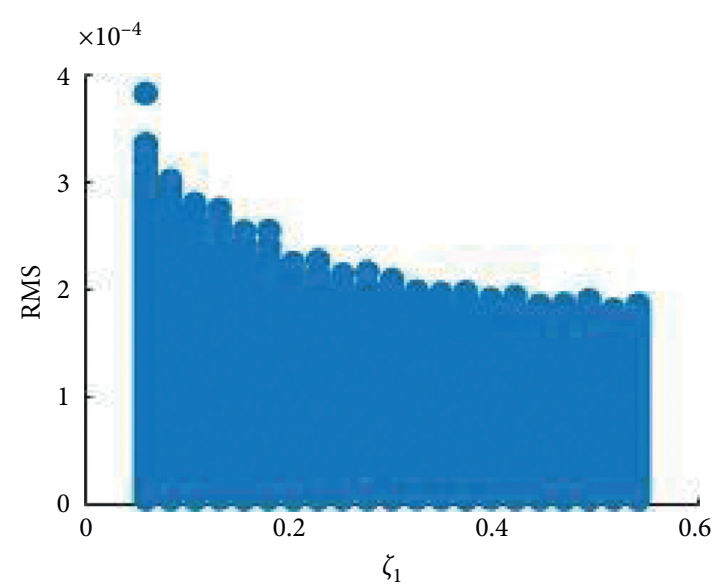

FIGURE 14: The distribution of calculation results corresponding to $\zeta 1$.

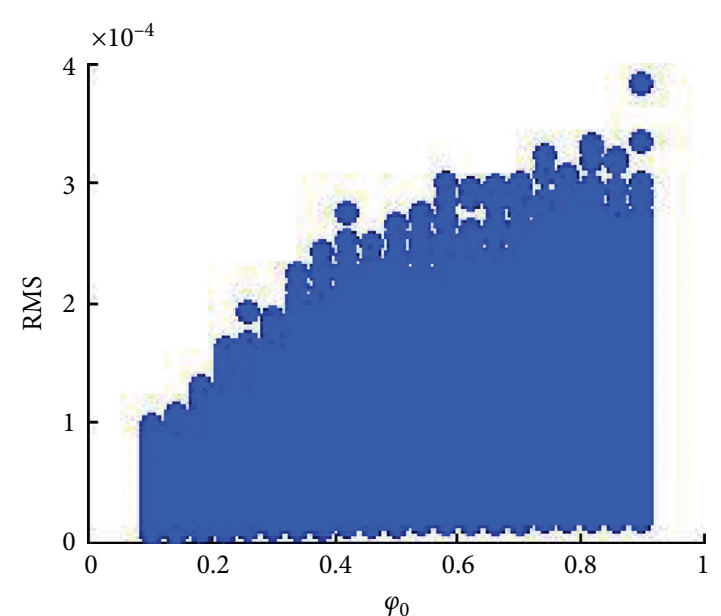

FIGURE 15: The distribution of calculation results corresponding to $\varphi 0$.

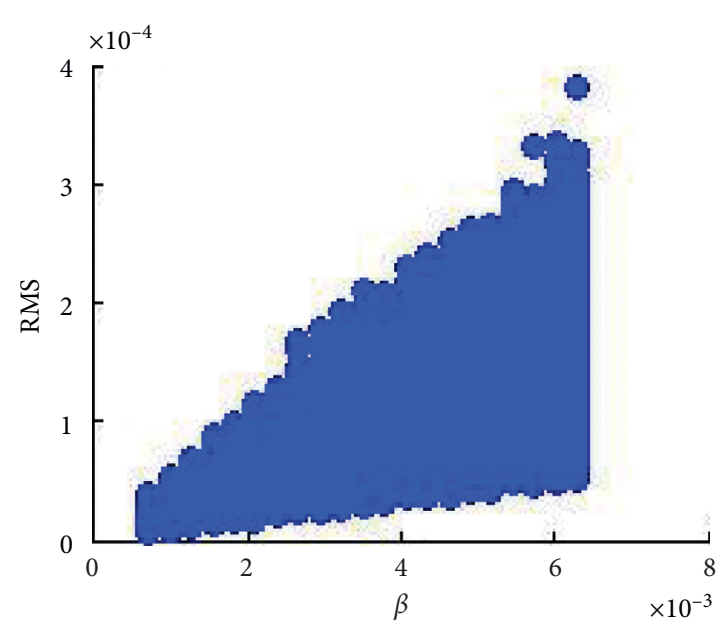

FIGURE 16: The distribution of calculation results corresponding to $\beta$.

parameters are as follows $\zeta 0=0.028, \zeta 1=0.54, \varphi 0=0.1$, $\beta=0.0007$, and the RMS value of excitation $\ddot{q}$ is 0.9997 , almost all of which are attenuated in comparison with $5.9102 \times 10^{-6}$, 
thus fully proving the effectiveness of Type I proposed in this paper.

\section{Conclusion}

In this paper, the biggest innovation is that two new types of SVRS with geometric nonlinear damping are proposed. For the two new types of SVRS, the paper firstly derives the motion differential equations of the two in a vertical direction, then carries out a comparative study on the vibration isolation performance of the two new types of SVRS under impact excitation and random excitation, respectively, chooses the optimal design scheme, and researches the influence rule of parameters of the chosen new type of SVRS on vibration isolation performance at last. This paper reaches the conclusion that the vibration isolation performance of diagonal damping is superior to that of lateral damping, and a new thought can be provided for the design of SVRS and other vibration reduction systems through the study result. Of course, the study in this paper also has its limitations. It rarely involves theoretical research on new SVRS, which will be our future research focus.

\section{Data Availability}

All the underlying data related to this article are available from the corresponding author upon request.

\section{Conflicts of Interest}

The authors declare that they have no conflicts of interest.

\section{References}

[1] Y. S. Zhang, "Vibration of tilted spring support," Journal of Mechanical Strength, vol. 14, no. 2, pp. 54-59, 1992.

[2] X. Xu, Analysis and Study on Suspension System with Package Cushioning, Xi'an University of Technology, Xian, China, 2005.

[3] X. Wu and L. J. Yang, "Natural vibration property of geometrically nonlinear vibration isolation system with suspension springs," Journal of Vibration and Shock, vol. 27, no. 11, pp. 71-72, 2008.

[4] X. Wu, Y. X. Luo, L. J. Yang et al., "Analysis on the vibration of shock absorber system with pendulum spring under foundation displacement excitation," Transactions of Beijing Institute of Technology, vol. 29, no. 12, pp. 1041-1043, 2009.

[5] L. Wang and A. J. Chen, "Shock characteristics of a suspension spring system under action of a rectangular pulse," Journal of Vibration and Shock, vol. 31, no. 11, pp. 142-144, 2012.

[6] L. Wang and A. J. Chen, "Study on shock characteristics of suspension spring packaging system," Packaging Engineering, vol. 32, no. 9, pp. 33-36, 2011.

[7] S. Shuang, D. Ningning, and C. Anjun, "Application of variational iteration method for dropping damage evaluation of the suspension spring packaging system," Abstract and Applied Analysis, vol. 2014, Article ID 385404, 7 pages, 2014.

[8] S. Shuang and C. Anjun, "Variational iteration method of dropping shock response for the suspension spring packaging system," Shock and Vibration, vol. 2015, Article ID 408674, 6 pages, 2015.
[9] X. C. Zhao and F. R. Bi, "Research on dynamical characteristics of vibration in suspension spring system," Journal of Machine Design, vol. 32, no. 1, pp. 43-46, 2015.

[10] X. C. Zhao and F. R. Bi, "Shock response characteristics of a suspended vibration reduction system," Journal of Vibration and Shock, vol. 33, no. 17, pp. 173-178, 2014.

[11] F. L. Kong, Research on the Dynamic Characteristics of Packaging System with Titled Support, Jiangnan University, Wuxi, China, 2012.

[12] N. Duan, M. Hao, and A. Chen, "Damage evaluation of critical components of tilted support spring nonlinear system under a rectangular pulse," Mathematical Problems in Engineering, vol. 2015, Article ID 910143, 9 pages, 2015.

[13] B. Jing and J. Xingiian, "Analysis and design of a novel and compact X-structured vibration isolation mount (X-Mount) with wider quasi-zero-stiffness range," Nonlinear Dynamics, vol. 101, no. 4, pp. 2195-2222, 2020.

[14] F. Xiao and J. Xingjian, "Human body inspired vibration isolation: beneficial nonlinear stiffness, nonlinear damping \& nonlinear inertia," . Mechanical Systems and Signal Processing, vol. 117, pp. 786-812, 2019.

[15] B. Jing and J. Xingjian, "Superior nonlinear passive damping characteristics of the bio-inspired limb-like or X-shaped structure," Mechanical Systems and Signal Processing, vol. 125, pp. 21-51, 2019.

[16] D. Guangxu, Z. Yahong, L. Yajun et al., "Enhanced isolation performance of a high-static-low-dynamic stiffness isolator with geometric nonlinear damping," Nonlinear Dynamics, vol. 93, no. 4, pp. 2339-2356, 2018.

[17] C. Chun, L. Shunming, W. Yong et al., "Force and displacement transmissibility of a quasi-zero stiffness vibration isolator with geometric nonlinear damping," Nonlinear Dynamics, vol. 87, no. 4, pp. 2267-2279, 2017.

[18] L. Wang, Research on Dynamic Characteristic of Suspension Cushioning Packaging System, Jiangnan University, Wuxi, China, 2012.

[19] Y. Shun and M. H. Zhang, "The nonlinear problem in the packaging dynamics," Packaging Engineering, vol. 16, no. 1, pp. 5-10, 1995.

[20] Y. Meng, X. Xinxi, S. Weihua et al., "The dynamic performance optimization for nonlinear vibration-reduction system of the tracked ambulance," Mechanical Engineering Science, vol. 229, no. 15, pp. 2719-2729, 2015.

[21] J. G. Yue, Z. G. Liu, Y. Q. Su et al., "Vulnerability study on large wind-turbine hydraulic control system," Chinese Journal of Construction Machinery, vol. 13, no. 4, pp. 299-306, 2015.

[22] Y. Meng, W. Meng, and H. Jiaqing, "The structure optimization for nonlinear vibration reduction system of the tracked ambulance," Journal of Vibroengineering, vol. 19, no. 1, pp. 577-591, 2017.

[23] A. B. I. Akmar, T. Lahmer, S. P. A. Bordas et al., "Uncertainty quantification of dry woven fabrics: a sensitivity analysis on material properties," Composite Structures, vol. 116, pp. 1-17, 2014. 